\title{
Refinamento do Conjunto Inicial de Resultados Utilizando Informações Contextuais para Recuperação Interativa de Imagens
}

\section{Luciano Araújo Dourado Filho ${ }^{\mathbf{1}}$; Rodrigo Tripodi Calumby ${ }^{2}$;}

1. Bolsista PIBIC/CNPq, Graduando em Engenharia da Computação, UEFS, e-mail: lucianoadfilho@gmail.com

2. Orientador, Departamento de Ciências Exatas, UEFS, e-mail: rtcalumby@uefs.br

PALAVRAS-CHAVE: Recuperação da Informação; Reranqueamento; Espaço Contextual.

\section{INTRODUÇÃO}

O avanço das tecnologias de captura e armazenamento de conteúdo digital proporciona um aumento na quantidade de dados (imagens, vídeos, e-mails, documentos, etc) que consequentemente demanda por desenvolvimento de técnicas para sua exploração tendo em vista sua grande quantidade e disponibilidade, em sistemas computacionais ao redor do mundo. Esses dados podem ser utilizados para fins diversos, como medicina, análise forense, estudo de biodiversidade, redes sociais, bibliotecas digitais, entre outros (Lew, 2001) e a eficácia em suas técnicas de exploração/recuperação podem tornar o acesso mais fácil e produtivo.

Quando trata-se de imagens, por exemplo, os principais paradigmas de busca consistem em levar em consideração informações textuais associadas (anotações, metadados, palavras-chave) para realizar sua recuperação para um usuário a partir de bancos de dados (Lieberman \& Rosenzweig, 2001). Outra abordagem comum se dá através da representação computacional das propriedades visuais de imagens (cor, forma e textura) e com base nisso computar similaridade entre imagens. As entidades responsáveis por realizar esse processo são denominadas descritores. Os sistemas computacionais de recuperação de imagens por conteúdo, podem, a partir de uma ou mais imagens de consulta, ordenar as imagens de sua base de

acordo com a similaridade em relação à imagem de consulta e retornar as mais parecidas para o usuário (Torres \& Falcão, 2006).

Um problema comum às duas abordagens citadas trata-se da noção de similaridade, que varia de acordo com a percepção de cada usuário e da discrepância entre a interpretação das características semânticas de uma imagem por um ser humano em relação à representação de suas propriedades por meio de uma máquina (gap semântico). Com intuito de minimizar o problema tenta-se combinar descritores visuais de modo a adaptar a busca às necessidades do usuário (Atrey et al., 2010). Outra possibilidade é permitir que o usuário interaja com o sistema, indicando a relevância das imagens no resultado o que possibilita ao sistema retornar outras similarmente relevantes (Ruthven \& Lalmas, 2010; Calumby et al., 2010). Com isso, à partir de técnicas de aprendizado de máquina é possível buscar por padrões que representem as necessidades do usuário.

A aplicação de técnicas de aprendizado de máquina com realimentação de relevância permitem ao usuário interagir com o sistema enquanto ele tenta aprender como atender às suas necessidades. Esse processo é realizado de maneira iterativa enquanto o usuário não se declarar satisfeito. O usuário alimenta o sistema ao longo das iterações quando indica a relevância dos itens exibidos, desta forma, o sistema tenta codificar quais propriedades visuais melhor caracterizam as imagens informadas como relevantes pelo usuário, portanto tende a retornar resultados mais relevantes, ao longo 
das iterações, em relação ao conjunto inicial. De forma intuitiva, se o conjunto inicial apresenta resultados não satisfatórios, a tendência é que o usuário não tenha como fornecer informação de qualidade para o sistema e assim os resultados consequentes também sejam insatisfatórios (Calumby et al., 2017).

Desta forma, têm-se desenvolvido técnicas de re-ranqueamento com o intuito de melhorar a eficácia de sistemas de recuperação de imagens. Exemplo disso é o método de re-ranqueamento baseado em espaços contextuais (Pedronette et al., 2014). A ideia geral é explorar as informações que as relações entre várias imagens podem prover. Deste modo, é possível redefinir o grau de similaridade entre duas imagens levando em consideração a similaridade de uma das imagens, em relação às suas vizinhas. Ou seja, é possível refinar as distâncias entre imagens levando em consideração o contexto em que uma delas estão inseridas.

Este trabalho realizou a avaliação do emprego desta técnica, com intuito de refinar o conjunto inicial de resultados de busca de imagens por conteúdo e analisar seu impacto ao longo das iterações.

\section{METODOLOGIA}

Sistemas comuns de recuperação de imagens por conteúdo computam distâncias entre imagens aos pares. Isso implica na perda de informação que as relações entre várias imagens podem revelar (Pedronette et al., 2014). Por isso, o autor propõe que seja possível explorar a vasta quantidade de informação que pode haver na relação entre uma imagem e suas imagens vizinhas, através do que autor denominou de Espaços Contextuais. Os Espaços Contextuais permitem analisar a relação que há entre uma imagem e seu Contexto (Pedronette et al., 2014). A partir disso, é possível refinar as distâncias entre imagens, levando em consideração as informações provenientes de seus contextos.

Seja A uma imagem, para cada uma das suas $\mathrm{K}$ imagens mais similares um espaço contextual é construído. Novas distâncias são computadas mesclando os espaços contextuais num único espaço definido por duas dimensões: $d_{i}$ e $d_{j}$ (Pedronette et al., 2014). Supondo que deseja-se redefinir a distância da imagem A para uma imagem B, a dimensão $d_{i}$ representa a distância entre A e B. Já a dimensão $d_{j}$ representa a distância dos vizinhos de $\mathrm{A}$ em relação à $\mathrm{B}$. A dimensão $\mathrm{d}_{\mathrm{j}}$ pode ser obtida por meio do somatório dos valores de distância dos vizinhos de $\mathrm{A}$ para $\mathrm{B}$, ponderado pela ordem de proximidade com A (imagens mais similares recebem pesos maiores). Já a dimensão $\mathrm{d}_{\mathrm{i}}$ é obtida por meio do valor de distância entre A e B, dividido pela quantidade de vizinhos. Por fim a distância é redefinida por meio da soma entre os componentes $d_{i}$ e $d_{j}$.

O método de Espaços Contextuais baseado em vizinhos mais próximos originalmente proposto em (Pedronette et al., 2014) sugere que o processo de reranqueamento seja feito de forma iterativa, porém trata-se de uma abordagem mais custosa computacionalmente em relação à forma não iterativa. Em sua forma não iterativa, os valores são calculados a cada iteração a partir dos valores da matriz de distâncias original e à cada iteração uma nova matriz de distâncias é gerada, diferentemente do modo iterativo, que a cada iteração os valores são reajustados a partir dos valores computados na iteração anterior e só no fim de sua execução uma nova matriz de distâncias é gerada. 
O método foi desenvolvido de modo não iterativo, visando ser avaliado por meio de experimentos com a base de imagens da ImageCLEF Photographic Retrieval Task (Thomas et al., 2009). Esta possui um conjunto heterogêneo de 20000 imagens com 180 imagens de consulta em que todas (imagens da base e imagens de consulta) apresentam descrição textual. Para isso, utilizou-se o arcabouço de realimentação de relevância (Calumby, 2010) para simular uma atividade de busca de imagens na web.

A avaliação foi realizada utilizando o gabarito fornecido junto à coleção e as mesmas métricas utilizadas na ImageCLEF Photographic Retrieval Task (Thomas et al., 2009) e trabalhos da literatura (Pedronette et al., 2014). Foram utilizados os descritores visuais: ccom, acc, las, bic, jac, qcch, bow e gch e os descritores textuais: jackard, cosseno, BM25, tfidf e dice. Os resultados foram comparados com os resultados originais (sem contexto) apresentados pelo arcabouço.

\section{RESULTADOS E DISCUSSÃO}

A Figura 1 apresenta os resultados de uma avaliação multimodal em que todos os descritores (visuais e textuais) foram empregados para realizar uma avaliação geral do método de baseado em contexto não iterativo. Dentre as métricas avaliadas optou-se por apresentar essa por denotar uma ideia geral do desempenho do arcabouço quando realizadas consultas com e sem contexto (método original). A partir dela é possível saber qual é a fração de itens relevantes à consulta dentro do conjunto de itens retornadas pelo sistema (Precision).

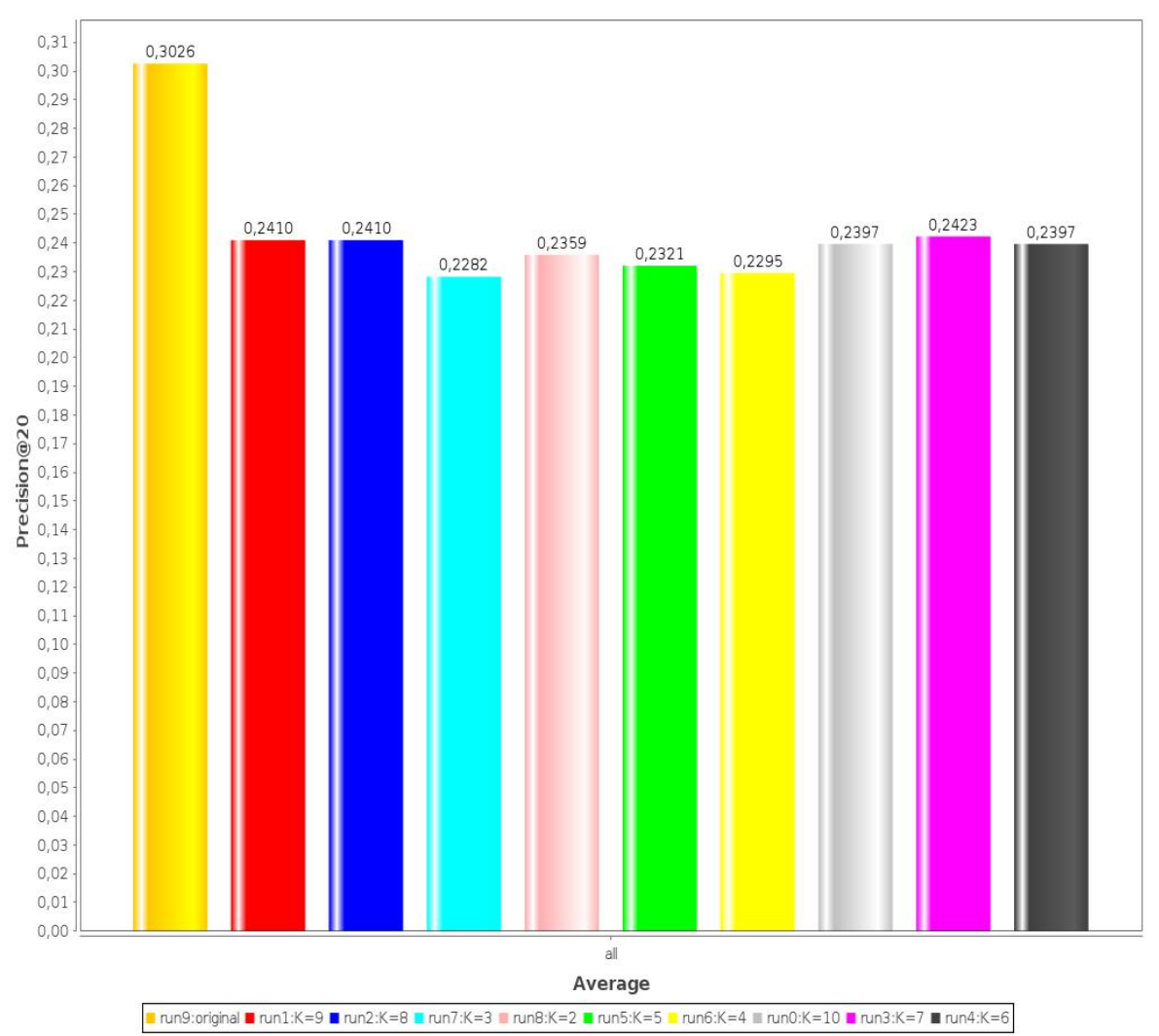

Figura 1: Valores de Precision para os respectivos parâmetros utilizados.

É possível notar que os resultados para o método contextual não iterativo encontrados apresentaram-se inferiores quando comparados com o original, independente da quantidade de vizinhos. Além disso, verificou-se também que o 
número de vizinhos considerados para o contexto (K) não causou mudanças consideráveis no valor de Precision.

Durante uma análise mais profunda sobre os resultados encontrados descobriuse que os valores de distância recalculados tenderam para um valor limite conforme a quantidade de vizinhos utilizados aumentava, por isso, acredita-se que tenha sido o fator que justifique a ausência de impacto expressivo do número de vizinhos na precisão dos resultados já que as distâncias entre as imagens não variaram significativamente para diferentes quantidade de vizinhos.

Acredita-se que tenha sido um fator que também justifique os resultados encontrados, o fato do método ter sido implementado de forma não iterativa afim de reduzir seu custo computacional, diferente da forma que Pedronette et al. (2014) propõe.

\section{Conclusão}

Deste modo, verificou-se que o método proposto na sua forma não iterativa apresentou resultados insatisfatórios para a base de dados utilizada. Para isso, implica realizar um estudo sobre sua forma iterativa, de modo a fazer uma análise mais profunda de seus resultados com o cenário proposto.

\section{REFERÊNCIAS}

A. THOMAS, C. PAUL, M. SANDERSON, and M. GRUBINGER. Overview of the ImageCLEFphoto 2008 photographic retrieval task. In Evaluating Systems for Multilingual and Multimodal Information Access, volume 5706 of Lecture Notes in Computer Science, pages 500-511. Springer Berlin / Heidelberg, 2009.

D. C. G. PEDRONETTE, R da S. TORRES e R. T. CALUMBY. Using contextual spaces for image re-rankingand rank aggregation. Multimed Tools Appl 69 (3), 689-716, 2014.

H. LIEBERMAN, E. ROSENZWEIG, and P. SINGH. Aria: An Agent for Annotating and Retrieving Images. IEEE Computer, 34(7),57-62, 2001.

I. RUTHVEN and M. LALMAS. A survey on the use of relevance feedback for information access systems. Knowl Eng. Rev. 18(2), 95-145 (2010).

M. S. LEW, editor. Principles of Visual Information Retrieval - Advances in Pattern Recognition. Springer-Verlag, London Berlin Heidelberg, 2001.

P. ATREY, M. HOSSAIN, A. El SADDIK, and M. KANKANHALLI. Multimodal fusion for multimedia analysis: a survey. Multimedia Systems, 16(6), 1-35 (2010). R. da S. TORRES, A. X. FALCÃO. Content-Based Image Retrieval: Theory and Applications. Revista de Informática Teórica e Aplicada 13(2), 161-185 (2006). R. T. CALUMBY . Recuperação Multimodal de Imagens Com Realimentação de Relevância Baseada em Programação Genética. Instituto de Computação da Universidade Estadual de Campinas. 2010.

R. T. CALUMBY, R. da. S. TORRES e M. A. GONÇALVES. Multimodal Retrieval with Relevance Feedback Based on Genetic Programming. Multimedia Tools and Applications, 69(3), 991-1019, 2014.

R. T. CALUMBY, R. da S. TORRES e M. A. GONÇALVES. Diversity-based Interactive Learning meets Multimodality. In Neurocomputing 259, 159-175, 2017. R.T. CALUMBY, R. da S. TORRES. Diversity-driven Learning for Multimodal Image Retrieval with Relevance feedback. In IEEE International Conference on Image Processing, pages 2197-2201, Paris, France, 2014. 